\title{
A Sampling Investigation of GPR Wave Propagation Velocity Data to Improve Migration Processing of Concrete Rebars
}

\author{
F. Tosti ${ }^{*}$, K. J. Munisami*, A. Sofroniou* ${ }^{*}$ A. M. Alani ${ }^{*}$ and F. Benedetto ${ }^{\dagger}$ \\ "School of Computing and Engineering, University of West London (UWL), London, United Kingdom \\ ${ }^{\dagger}$ Signal Processing for Telecommunication and Economics Lab., University of Roma Tre, Rome, Italy \\ Email: Fabio.Tosti@uwl.ac.uk; Kevin.Munisami@uwl.ac.uk; Anastasia.Sofroniou@uwl.ac.uk; Amir.Alani@uwl.ac.uk; \\ francesco.benedetto@uniroma3.it
}

\begin{abstract}
In this study, a demonstration of the potential of ground-penetrating radar (GPR) for improving location of rebars is given. To this purpose, a high-frequency GPR system with a $2000 \mathrm{MHz}$ dual-polarised antenna (HH/VV polarisations) has been used to collect data on a reinforced concrete floor of dimensions $1 \mathrm{~m} \times 0.80 \mathrm{~m}$ (longitudinal and transversal acquisitions). The high-dense grid mesh of rebars and the use of the hyperbola fitting method allowed for the acquisition of a dataset of wave propagation velocity values. Hence, an analysis of the statistic distribution of propagation velocity values was carried out to assess the data dispersion throughout the area. A data sampling approach was then proposed and velocity values were sampled at different percentages and in an evenlydistributed manner throughout the inspected area. Corresponding values of velocity were therefore used for data migration purposes and $\mathrm{C}$-scan maps were produced as a combination of longitudinal/transversal acquisitions and $\mathrm{HH} / \mathrm{VV}$ polarisations. The optimum sampling rate of wave propagation velocity was then assessed by way of comparison of the migrated maps.
\end{abstract}

Keywords-GPR; data sampling; hyperbola fitting; data migration; rebars location

\section{INTRODUCTION}

Investigation of rebars in reinforced concrete is a matter of high interest in civil engineering. Reinforced concrete has been widely used for over a century as a high-performance construction material, although it is subject to damage and deterioration for a variety of different and complex reasons [1]. Hence, a large number of tests and routine assessments are usually required for this material [2]. To this effect, a variety of destructive and non-destructive testing (NDT) methods have been used to identify specific features of rebars.

NDT methods are gaining momentum in this area of research [3]. Ultrasonic sensors are used to assess dimensions of the rebars as well as for damage location purposes (e.g., voids and cracks) [4]; reinforcement location and assessment of corrosion are pursued using electromagnetic (EM) methods, such as radiography [5], eddy current sensors [6] and impedance tomography. Chemical-based techniques, thermography and impedance tomography [7] are widely employed for detection of moisture. Within this context, the ground-penetrating radar (GPR) NDT method has proven to be suitable for many of the above application areas. In more detail, GPR systems with high-frequency antennas are extensively used on concrete structures, and several GPR-based algorithms for effective assessment of corrosion and moisture infiltration have been developed in the literature [8]. Research is nowadays being oriented towards the assessment of the size of rebars [9]. One of the most common applications of GPR in reinforced concrete relates with the location of rebars [10] and the estimation of the concrete cover depth [9]. Accuracy of the above estimations are improved using dedicated data processing algorithms, e.g., migration [11], and data interpretation methods, e.g., hyperbola fitting [12]. In this framework, although a good degree of accuracy can be achieved with the available commercial software, a higher precision might be necessary for quality control and routine inspection purposes. To speed up the data processing, a few values of velocity of propagation are usually randomly extracted by fitting the reflections from the clearest hyperbola shapes in the B-scan. The average velocity is then assigned to the whole area to obtain a migrated map. In this regard, although relating an average velocity value to a whole investigation area might not work for large and complex environments (i.e., investigation sites with high horizontal and vertical variations of wave propagation velocity, which are mostly unknown), it is more functional for the location of rebars in relatively small areas made of reinforced concrete. This process can be improved by sampling a consistent number of targets for an estimation of velocity by hyperbola fitting, such that a more representative average value is assigned to the area for data migration purposes.

\section{AIM AND OBJECTIVES}

The main aim of this study is to comprehend the effect of different data sampling percentages on the estimation of a propagation velocity value representative of a reinforcedconcrete-paved area with a high-dense grid mesh of rebars.

Estimation of this parameter is therefore used for data migration purposes in order to produce a viable C-scan map for a more accurate location of concrete rebars. 
To achieve this Aim, the following objectives are identified:

- to create a 2-D matrix of wave propagation velocity values estimated by hyperbola fitting of the reflections from the rebars over the whole inspected area;

- to analyse the statistical distribution of the estimated propagation velocity values for assessing the data dispersion throughout the area;

- to develop a data sampling methodology in order to extract a value of velocity of propagation representative of the entire area. This is pursued by way of comparison between the tomographic maps migrated at different sampling percentages (combination of longitudinal/transversal acquisitions and horizontal (HH)/vertical (VV) polarisations).

\section{METHODOLOGY}

The methodology is divided into four main stages. Data acquisition is first carried out. A second main stage focuses on the estimation of the wave propagation velocity values using the hyperbola fitting method. This allows to create a 2-D matrix of data throughout the area inspected. A third stage is then developed to analyse the statistical distribution of the above estimates and assess the dispersion of the data. Finally, a data sampling methodology is proposed to extract a representative wave propagation velocity value for data migration purposes.

\section{A. Data Acquisition}

Data were collected on a reinforced-concrete-paved area (rebar density of $\sim 5$ rebars $/ \mathrm{m}^{2}$ ) with dimensions of $1 \mathrm{~m} \times 0.80 \mathrm{~m}$ (Fig. 1). The IDS Aladdin GPR system equipped with a dualpolarised antenna of $2000 \mathrm{MHz}$ central frequency was used for testing purposes. The antenna configuration allowed to collect two different sets of data at the same time along a single scan line, as per the $\mathrm{HH}$ and VV polarisations. GPR signals were acquired with a horizontal resolution of $1 \mathrm{~cm}$, using a time window of $32 \mathrm{~ns}$ and 512 samples. Both longitudinal and transversal scans were carried out, with a scan spacing of 5 $\mathrm{cm}$.

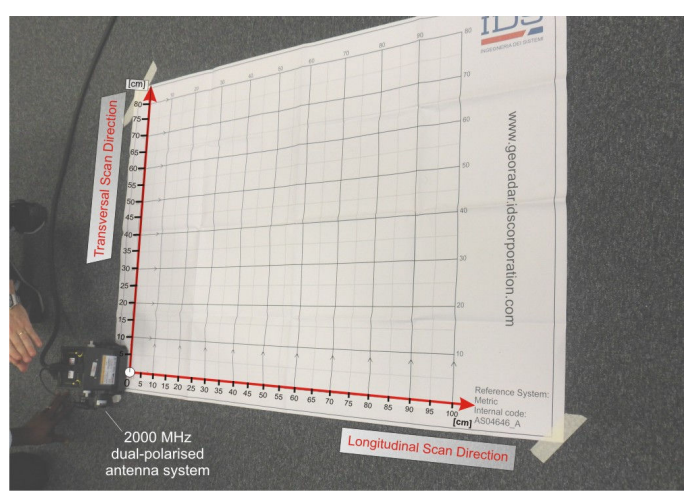

Fig. 1. Scanning grid and $2000 \mathrm{MHz}$ dual polarised antenna system used for the acquisitions.

\section{B. Wave Velocity of Propagation Data Matrix by Hyperbola Fitting Method}

In view of the high-dense grid mesh of rebars and the relatively small scan sapcing, the hyperbola fitting method [13] was used to assess wave propagation velocity data throughout the whole area investigated. In this method, the wave velocity $v$ is proportional to the angle $\alpha$ between the hyperbola asymptotes, according to the following relationship:

$$
v=2 \operatorname{tg} \alpha
$$

Several studies in the literature have proven the dependency of $v$ on the vertex coordinates, the target radius and the time delay of signal reflection. An example of hyperbola fitting for one of the rebars investigated in this study is reported in Fig. 2.

From the GPR acquisitions, it was possible to detect two main layers of rebars. In more detail, the area inspected included $n=5$ upper lines of rebar (R1, R2, R3, R4 and R5 in Fig. 2) overlying $l=5$ lower rebar lines (RL1, RL2, RL3, RL4 and RL5 in Fig. 2). For the purposes of this study, only the upper rebars were taken into account. Hence, considering a scan spacing of $5 \mathrm{~cm}, m=17$ longitudinal scans of $1 \mathrm{~m}$ length were collected across the $80 \mathrm{~cm}$-wide transversal dimension of the inspection area. This turned out to provide a $n \times m=85$ matrix of rebar points and, hence, a matrix $V$ of wave velocity of propagation data.

\section{Statistical Distribution Analysis}

An analysis of the statistical distribution of the dataset of propagation velocity values $v_{i, j, 100}^{[m, n]}$ is carried out to assess the data dispersion throughout the investigated area. The average value of velocity of propagation of the entire population $\overline{v_{i, j, 100}}$ (value averaged over $k=100 \%$ of the $m \times n$ data in the propagation velocity matrix) is taken as a reference for each combination of $i^{\text {th }}$ scan direction and $j^{\text {th }}$ antenna orientation in the calculation of residuals. Hence, the percentage residual at a generic position $[m, n]$ in the matrix is calculated as follows:

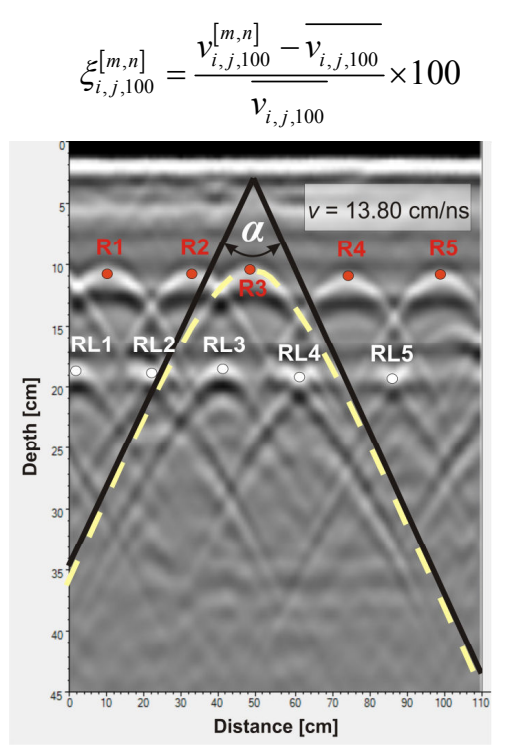

Fig. 2. Estimation of wave propagation velocity on a longitudinal scan $(\mathrm{HH}$ polarisation) by the hyperbola fitting method and typical arrangement of rebars within the inspection area. 
A matrix $R$ of residuals $\xi_{i, j, 100}^{[m, n]}$ sized $m \times n$ can be therefore computed by working out the values of $v_{i, j, 100}^{[m, n]}$ in (1). To assess the dispersion of propagation velocity values $v_{i, j, 100}^{[m, n]}$ against the reference $\overline{v_{i, j, 100}}$, the $m^{\text {th }}$ average residual $\overline{\xi_{i, j, 100}^{[\hat{m}, n]}}$ is defined along the $m=17$ scanning lines as follows:

$$
\overline{\xi_{i, j, 100}^{[\hat{m}, n]}}=\sum_{n=1}^{5} \xi_{i, j, 100}^{[m, n]} \quad \forall m \in R
$$

and compared to the average residual of the entire matrix population $\overline{\xi_{i, j, 100}}$ (value averaged over $k=100 \%$ of the $m \times n$ residuals $\xi_{i, j, 100}^{[m, n]}$ in $R$ ). Hence, data dispersion from this reference value is interpreted as a measure of nonhomogeneous distribution of the propagation velocity throughout the inspected subsurface area. Also, this analysis is useful to identify potential misalignments of the rebars along the scanning line. The frequency density distributions of residuals for $i$ ) the entire matrix population $\xi_{i, j, 100}^{[m \times n]}$ and ii) each $n^{\text {th }}$ rebar $\overline{\xi_{i, j, 100}^{[m, \hat{n}]}}$ is also defined, being this latter expressed as follows:

$$
\overline{\xi_{i, j, 100}^{[m, \hat{n}]}}=\sum_{m=1}^{17} \xi_{i, j, 100}^{[m, n]} \quad \forall n \in R
$$

\section{Data Sampling and Migration}

A number of different $k^{\text {th }}$ percentages of $v_{i, j, k}^{[m, n]}$ values (with $k=5,10,15,20,30,50,50$ inverted) is sampled over the $n \times m$ data in the velocity of propagation matrix $V$. Sampling is developed to ensure an evenly distribution of data across the longitudinal and transversal directions of the area (Fig. 4). Sampling configuration ranges from diagonal (i.e., case of smaller percentages (Fig. 4(a)) to a chessboard-like arrangement at $k=50$ and 50inverted (Fig. 4(f-g)). The optimum sampling rate of wave propagation velocity was then assessed by way of comparison of the migrated maps.
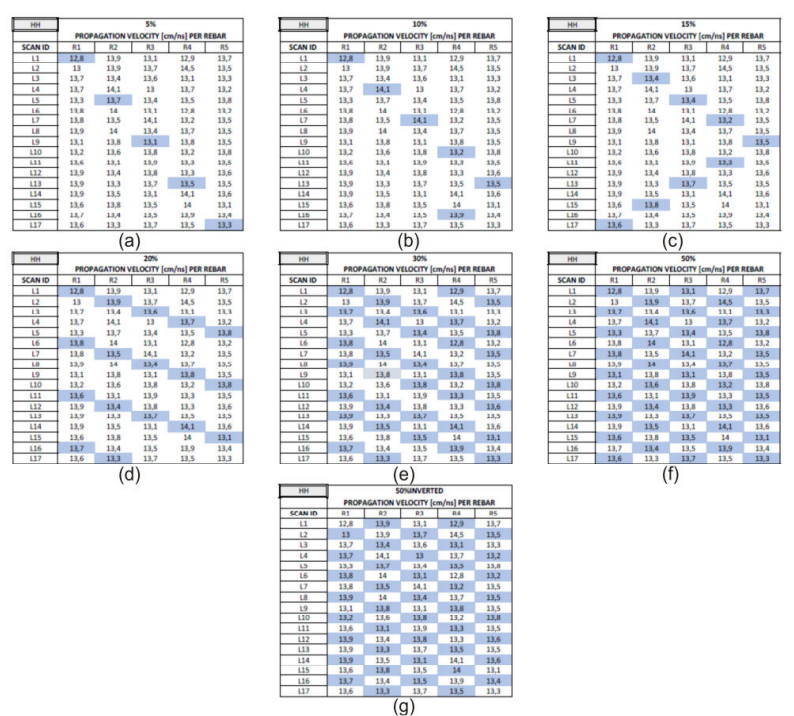

Fig. 3. Data sampling (blue cells) of the $k^{\text {th }}$ percentages of $v_{i, j, k}^{[n, m]}$ values in $V$ (with $k=5$ (a), 10 (b), 15 (c), 20 (d), 30 (e), 50 (f) and 50inverted (g)).

\section{RESUltS AND SHORT DisCUSSION}

\section{A. Statistical Distribution Analysis}

Table I shows the main statistics from the wave velocity of propagation data matrices, as per the combinations of $i(\mathrm{~L}, \mathrm{~T})$ and $j(\mathrm{HH}, \mathrm{VV})$. Overall, the average value of propagation velocity $\overline{v_{i, j, 100}}$ varies between 13.43 and $14.22 \mathrm{~cm} / \mathrm{ns}$ across the four $i^{\text {th }}, j^{\text {th }}$ combinations. The maximum standard deviation $\left(\sigma_{v_{i, j, 100}}=1.03 \mathrm{~cm} / \mathrm{ns}\right)$ and standard error $\left(\sigma_{\overline{v_{i, j, 1,100}}}=0.11 \mathrm{~cm} / \mathrm{ns}\right)$ are observed in the case of longitudinal scan and $\mathrm{VV}$ polarisation.

In regard to the statistics for the residual distributions, for sake of brevity only the results on the longitudinal scan/HH polarisation are reported. Fig. 4 and 5 show the distribution of the average $\overline{\xi_{i, j, 100}^{[\hat{m}, n]}}$ and the generic residual $\xi_{i, j, 100}^{[m, n]}$, respectively, from the scan position $j=1(0 \mathrm{~cm}$ on the Tscan axis) to $j=17$ (80 $\mathrm{cm}$ on the Tscan axis), sorted by rebar number $n$. From the analysis of $\overline{\xi_{i, j, 100}^{[\hat{m}, n]}}$ (Fig. 4), a larger dispersion is observed from $j=1(0 \mathrm{~cm}$ on the Tscan axis) to $j$ $=7(35 \mathrm{~cm}$ on the Tscan axis), as opposed to the rest of the scan. From Fig. 5, it can be noticed that the first (R1) and the forth (R4) lines of rebar have the largest dispersion among the 5 rebar lines investigated. On the contrary, the fifth line of rebar (R5) seems to provide less variation over $\overline{v_{i, j, 100}}$. This is confirmed by the percentage residuals $\overline{\xi_{i, j, 100}^{[m, \hat{n}]}}$ at each $n^{\text {th }}$ rebar in Table II, where the rebar line R5 has the lowest statistics.

TABLE I. MAIN STATISTICS FROM THE WAVE VELOCITY OF PROPAGATION DATA MATRICES

\begin{tabular}{|c|c|c|c|c|}
\hline \multirow{2}{*}{$\begin{array}{c}\text { Statistic } \\
\text { Parameter } \\
{[\mathbf{c m} / \mathbf{n s}]}\end{array}$} & \multicolumn{2}{|c|}{$\begin{array}{c}\text { Longitudinal Scan } \\
(\boldsymbol{i}=\mathbf{L})\end{array}$} & \multicolumn{2}{c|}{$\begin{array}{c}\text { Transversal Scan } \\
(\boldsymbol{i}=\mathbf{T})\end{array}$} \\
\cline { 2 - 5 } & $\begin{array}{c}\boldsymbol{H} \boldsymbol{H} \text { Polar. } \\
(\boldsymbol{j}=\boldsymbol{H H})\end{array}$ & $\begin{array}{c}\boldsymbol{V} \boldsymbol{V} \text { Polar. } \\
(\boldsymbol{j}=\boldsymbol{V} \boldsymbol{V})\end{array}$ & $\begin{array}{c}\boldsymbol{H} \boldsymbol{H} \text { Polar. } \\
(\boldsymbol{j}=\boldsymbol{H H})\end{array}$ & $\begin{array}{c}\boldsymbol{V} \boldsymbol{V} \text { Polar. } \\
(\boldsymbol{j}=\boldsymbol{V} \boldsymbol{V})\end{array}$ \\
\hline$\overline{v_{i, j, 100}}$ & 13.54 & 13.58 & 13.43 & 14.22 \\
\hline$\sigma_{\overline{v_{i, j, 100}}}$ & 0.04 & 0.11 & 0.10 & 0.06 \\
\hline$\sigma_{v_{i, j, 100}}$ & 0.33 & 1.03 & 0.78 & 0.46 \\
\hline$v_{i, j, 100}^{\min }$ & 12.80 & 9.10 & 9.90 & 13.50 \\
\hline$v_{i, j, 100}^{M A X}$ & 14.50 & 14.80 & 14.10 & 15.40 \\
\hline
\end{tabular}

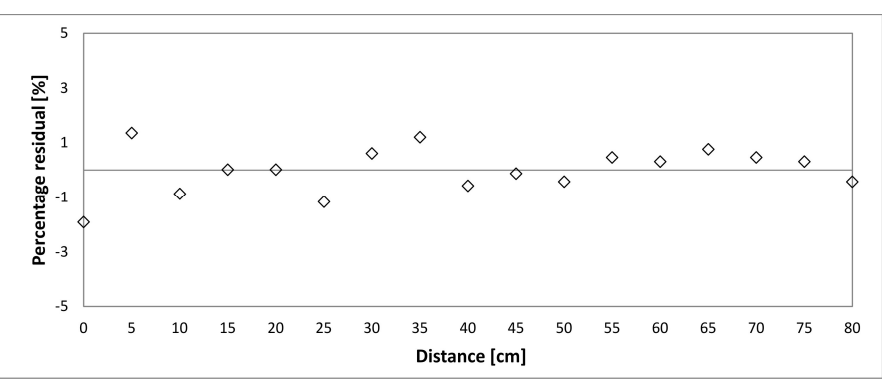

Fig. 4. Trend of the average percentage residual $\overline{\xi_{i, j, 100}^{[\hat{m}, n]}}$ along the $m=17$ scan lines. 


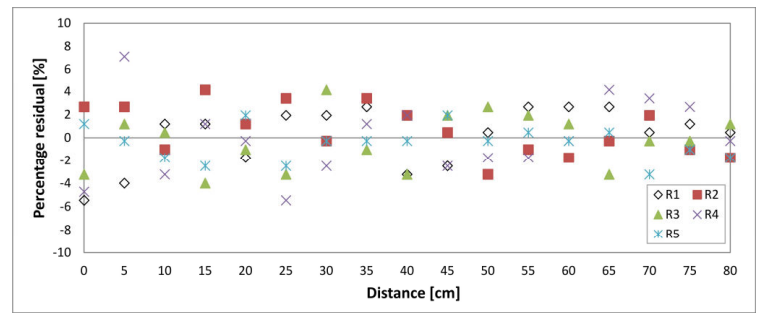

Fig. 5. Trend of the percentage residual $\xi_{i, j, 100}^{[m, n]}$ along the $m=17$ scan lines.

TABLE II. Main Statistics Regarding the Percentage Residuals $\overline{\xi_{i, j, 100}^{[m, \hat{n}]}}$ AT EACH REBAR

\begin{tabular}{|c|c|c|c|c|c|}
\hline \multirow{2}{*}{$\begin{array}{c}\text { Statistic } \\
\text { Parameter } \\
{[\%]}\end{array}$} & \multicolumn{5}{|c|}{$\begin{array}{c}\text { Longitudinal Scan }(i=\mathbf{L}) \\
\text { HH Polar. }(i=\mathrm{HH})\end{array}$} \\
\hline & $\begin{array}{c}R 1 \\
(n=1)\end{array}$ & $\begin{array}{c}R 2 \\
(n=2)\end{array}$ & $\begin{array}{c}R 3 \\
(n=3)\end{array}$ & $\begin{array}{c}R 4 \\
(n=4)\end{array}$ & $\begin{array}{c}R 5 \\
(n=5)\end{array}$ \\
\hline$\overline{\xi_{i, j, 100}^{[m, \hat{n}]}}$ & 0.16 & 0.68 & -0.28 & -0.06 & -0.50 \\
\hline$\sigma_{\overline{\left.\xi_{i, j, 100}^{m, n}\right]}}$ & 0.62 & 0.53 & 0.59 & 0.80 & 0.36 \\
\hline$\sigma_{\xi_{i m, \hat{n}, 100}}$ & 2.57 & 2.18 & 2.44 & 3.30 & 1.49 \\
\hline$\xi_{i, j, 100}^{[m, \hat{n}] \min }$ & -5.45 & -3.23 & -3.97 & -5.45 & -3.23 \\
\hline$\xi_{i, j, 100}^{[m, \hat{n}] M A X}$ & 2.68 & 4.15 & 4.15 & 7.11 & 1.94 \\
\hline
\end{tabular}

\section{B. Data Sampling and Migration}

Data migration was carried out using the average values of velocity of propagation $\overline{v_{i, j, k}^{[m \times n]}}$ at the aforementioned $k^{\text {th }}$ percentages of sampling. To assess the viability of the sampling approach and percentage of data used, the corresponding migrated C-scan tomographic maps were compared. Fig. 6 shows the C-scan maps (longitudinal scan; $\mathrm{HH}$ polarisation) of the inspected area after running data migration at $k=10, k=30$, and $k=100$, and collected at a depth $z=13 \mathrm{~cm}$. It can be seen that the use of a small percentage of data samples (i.e., Fig. 6a), was not sufficient to reproduce a viable configuration of rebars, as it is shown in Fig. 6c. On the contrary, the spatial sampling and data percentage used in the case of $k=30$ (i.e., Fig. 6b), seems to provide a more consistent output.

\section{CONCLUSION}

This paper presents a data sampling investigation for the estimation of a propagation velocity value representative of a reinforced-concrete-paved area with a high-dense grid mesh of rebars $\left(\sim 5\right.$ rebars $\left./ \mathrm{m}^{2}\right)$. To this purpose, a high-frequency GPR system with a $2000 \mathrm{MHz}$ dual-polarised antenna (HH/VV) was used and longitudinal and transversal scans were collected. The research methodology is divided into four main stages, including the data acquisition, use of the hyperbola fitting method, a statistical distribution analysis of the velocity values and residuals, and a data sampling methodology and migration.

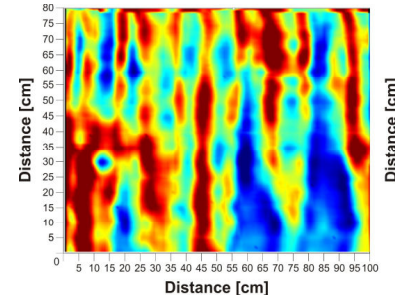

(a)

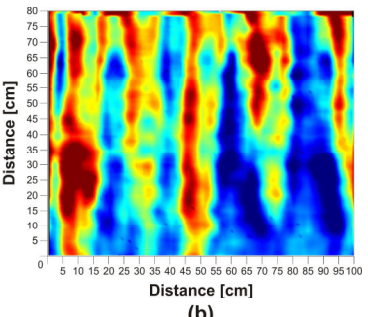

(b)

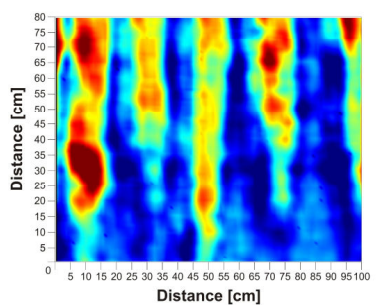

(c)

Fig. 6. C-scan maps of the inspected area after data migration at different $k^{\text {th }}$ percentages of sampling. $k=10(\mathrm{a}), k=30(\mathrm{~b})$ and $k=100(\mathrm{c})$.

Results have proven that the horizontal polarisation is usually more stable for the estimation of propagation velocity values by hyperbola fitting. In addition, it has been shown that a more accurate visualisation of the rebars can be obtained using a $5 \mathrm{~cm}$ scan spacing and information from at least the $30 \%$ of targets in a high-dense grid mesh arrangement of rebars.

\section{REFERENCES}

[1] Z. Guo, Principles of Reinforced Concrete, Elsevier, The Netherl., 2014.

[2] B. Szymanik, P.K. Frankowski, T. Chady and C. R. A. J. Chelliah, "Detection and inspection of steel bars in reinforced concrete structures using active infrared thermography with microwave excitation and eddy current sensors" Sensors, 16, 234, 2016.

[3] C. Maierhofer, H. W. Reinhardt and G. Dobmann, "Non-Destructive Evaluation of Reinforced Concrete Structures; Woodhead Publishing CRC Press: Cambridge, UK, 2010.

[4] M. Schickert, "Ultrasonic NDE of concrete". In Proceedings of the 2002 IEEE Ultrasonics Symposium, Munich, Germany, 8-11 2003; 739-748.

[5] H. Martz, D. Scheberk, G. Roberson and P. Monteiro, "Computerized tomography analysis of reinforced concrete," ACI Mater. J., vol. 90, pp. 259-264, 1993.

[6] T. Chady and M. Enokizono, "Multi-frequency exciting and spectrogram based ECT method," J. Magn. Magn. Mater. 215-216, p.700-703, 2000.

[7] P. Berowski, S. F. Filipowicz, J. Sikora and S. Wójtowicz, "Determining location of moisture area of the wall by $3 \mathrm{~d}$ electrical impedance tomography," In Proc. of the 4thWorld Congress on Industrial Process Tomography, Aizu, Japan, 2-5 Sept. 2005.

[8] C. Maierhofer, Nondestructive evaluation of concrete infrastructure with ground penetrating radar. ASCE J. Mater. Civil Eng.15, 287-297, 2003.

[9] V. Utsi and E. Utsi, "Measurement of reinforcement bar depths and diameters in concrete," In Proc. of the Tenth International Conference Ground Penetrating Radar, GPR 2004 vol. 2, 2004, pp. 659-662

[10] F. Soldovieri, R. Persico, E. Utsi and V. Utsi, "The application of inverse scattering techniques with ground penetrating radar to the problem of rebar location in concrete,"NDT E Int., 39(7),602-607, 2006.

[11] C.W. Chang, C. H. Lin and H. S. Lien, "Measurement radius of reinforcing steel bar in concrete using digital image GPR," Constr. Build. Mater., vol 23 (2), 2009.

[12] S. Tillard and J.C. Dubois, "Analysis of GPR data: wave propagation velocity determination,” J. Appl. Geophys., vol. 33, pp. 77-91, 1995.

[13] S. Shihab and W. Al-Nuaimy, "Radius estimation for cylindrical objects detected by ground penetrating radar," Subs. Sens. Technol. Appl., 6, pp.

151-166, 